\title{
OPTIMIZATION OF SODIUM LIGNOSULFONATE TREATMENT ON NYLON FABRIC USING BOX-BEHNKEN RESPONSE SURFACE DESIGN FOR UV PROTECTION
}

\author{
Arun Kumar Singh Gangwar ${ }^{1, *}$, D B Shakyawar ${ }^{2,3}$, Mukesh Kumar Singh ${ }^{1}$, Prashant Vishnoi ${ }^{1}$ and Seiko Jose ${ }^{3}$ \\ 1 Uttar Pradesh Textile Technology Institute, Kanpur-208001, India \\ 2 ICAR-National Institute of Natural Fibre Engineering and Technology, 12,Regent Park, Kolkata-700040, West Bengal, India \\ 3ICAR-Central Sheep and Wool Research Institute, Avikanagar 304501, India \\ ${ }^{*}$ Corresponding author. E-mail: arungangwar@rediffmail.com
}



Abstract:

The effect of sodium lignosulfonate (LS) treatment on nylon fabric for enhancing its ultraviolet protection ability has been studied. Various concentrations of LS were applied on nylon fabric using the exhaust method and the treatment was optimized using Box-Behnken response surface design. The ultraviolet protection factor (UPF) is achieved, as high as 62.13 with one such LS-treated nylon fabric. The LS-treated nylon fabrics were characterized using FTIR, FESEM, Energy dispersive X-ray (EDX), and Thermo-gravimetric analysis (TGA) instruments. The UPF and color-strength (K/S) values are significantly increased with an increase in the concentration of LS without any loss of tensile properties and thermal stability. The LS treatment has excellent wash fastness.

\section{Keywords:}

Sodium lignosulfonate, kraft lignin, ultraviolet protection, nylon 6, luster, protective clothing

\section{Introduction}

Ceaseless increase in the emission of greenhouse gases across the globe is the prime cause of deterioration in the ozone layer, and as a consequence of this environmental damage, human beings have come under the danger zone of ultraviolet radiation. Prolonged exposure of human beings to sunlight causes various dermatological diseases such as photo-aging, mutation of skin cells, and even skin cancer [1]. Ultraviolet Protection Factor (UPF) is a kind of transmission analysis of ultraviolet radiation (UVR) that quantifies exactly how much quantity of it is permitted across the materials. UPF gauges a fabric's effectiveness against both UVA and UVB radiation. The most important factors influencing UPF of fabric are fiber characteristics, fabric porosity, thickness, and dyeing and finishing chemicals [2,3]. Many dyes absorb UV, which helps to reduce UV exposure. Darker colors tend to absorb more UV than lighter colors, including whites and pastels, but bright colors such as red can also substantially absorb UV rays [4]. White fabric having optical whitening agents strongly absorbs UVR [5]. Radiation absorbed by a dye, pigment, delustrant, and UV absorber in the fabric restricts the passage of UVR through the skin, resulting in better sun protection.

Nylon 6 is one of the most useful synthetic fibers with applications produced in the form of filament and staple fiber. It has good strength, heat, and chemical resistance. However, it cannot provide protection from UV radiation. Therefore, suitable UV absorbers are used to improve the UPF of nylon fabrics. Saravanan (2007) reported that the application of $1.50 \% \mathrm{TiO}_{2}$ on microfine nylon fabrics provides good resistance towards UV radiation and is capable of giving UPF > 50 [6]. Till date, studies have been conducted on the modification of textile fibers, including nylon, with various inorganic substances, dye molecules, and pigments. There is a dearth of studies available 
in the literature on the modification of nylon fabrics with organic waste material such as sodium lignosulfonate (LS).

Sodium lignosulphonate is a brown color powder obtained as a by-product from pulp and paper mills. During pulping, the lignin of the cellulosic biomass is dissolved in high alkali concentration (black liquor) and precipitated later as LS. It is having a molecular mass of $534 \mathrm{~g}^{\circ} \mathrm{mol}^{-1}$. It has a wide range of industrial applications viz., pesticides, surfactants, additives in oil drilling, metal adsorbents, stabilizers in colloidal suspensions, plasticizers, and dispersants [7-9]. Qana et al. (2020) studied the use of lignin as a raw material/reactive component in a UV curable system and the effect of chemical modification of lignin on the film properties produced by UV radiation [10]. The existence of the abundance of the aromatic skeleton and hydrogen bonds helps LS to absorb ultraviolet radiation [11, 12]. Due to the presence of the sulfonated group, LSs are anionically charged and water-soluble [13]. LS is an agricultural bio waste, water-soluble substance. If it can be used for the finishing of textile materials for functional applications such as UV protection, a suitable alternative will be established for the bio-finishing of textiles.

In this study, LS solutions of various concentrations were prepared and treatment on nylon 6 fabrics was done by an exhaustion method. The process parameters of the treatment, i.e., the concentration of LS, temperature, and $\mathrm{pH}$ of LS solution were optimized using Box-Behnken response surface design. The treated fabrics were characterized by FTIR, FESEM, Energy dispersive X-ray (EDX), thermo-gravimetric analysis (TGA) techniques. The LS-treated nylon fabric samples were evaluated for UPF, K/S, tensile properties, and luster in detail.

\section{Experimental}

\subsection{Materials}

Nylon filament having a linear density of 149 deniers was procured from M/s JCT Ltd. Hoshiarpur, Punjab, India. Sodium lignosulphonate power was procured from M/s Lignotech, Umkomass, South Africa.

\subsection{Preparation of nylon fabric and treatment with LS}

Nylon filament was woven into the $2 / 2 \mathrm{t}$ will fabric of $90.8 \mathrm{~g} \times \mathrm{m}^{-2}$ areal density with 26 threads per $\mathrm{cm}$ in the warp and 31 threads per $\mathrm{cm}$ in a weft direction using a loom. The fabric was woven with the suitable thread density for the preparation of the fabric of the above areal density with the available reed on the loom.

The fabric was scoured with $5 \mathrm{~g} \times \mathrm{L}^{-1}$ non-ionic detergent for $30 \mathrm{~min}$ at $60^{\circ} \mathrm{C}$ before LS treatment. Various concentrations of LS solution (10\%, $15 \%$, and $20 \%$ ) were prepared in an aqueous medium and the nylon fabric was treated in an infrared heating chamber for 30 min by varying the temperature and $\mathrm{pH}$.

\section{$\underline{2.3 \text { Design of experiment }}$}

The LS concentrations, treatment temperature, and $\mathrm{pH}$ were taken as independent variables. The levels of these parameters are shown in Table 1. The levels are selected based on a preliminary experiment where satisfactory results were obtained. The coating of LS over the textile surface depends on the temperature and $\mathrm{pH}$ of the solution. Depending on the $\mathrm{pH}$ of the treatment solution, the color of LS coating varies, which was evidenced by Ultraviolet-visible (UV-Vis) spectroscopy analysis. The color of the sample may ultimately affect the UPF of the treated fabrics. The difference in the color of LS at different $\mathrm{pH}$ is shown in Figure 1(a). It is observed from the figure that the color of LS is sensitive to change in $\mathrm{pH}$. The color of the LS solution is pale brown in a slightly acidic and neutral $\mathrm{pH}$. However, it turned dark brown in an alkaline $\mathrm{pH}$. The main cause of the dark brown color of lignin is the groups of quinonoid structures. As mentioned earlier, the catechol structure in LS is easy to oxidize to quinonoid structures, which render the lignin strongly colored [14]. Figure 1 (b) shows the UV-Vis spectra of the LS solution. The solution shows the absorbency at $280 \mathrm{~nm}$. The peak is due to the content of guaiacyl compound. Similar observations have been reported by Yang et al. (2015) [14].
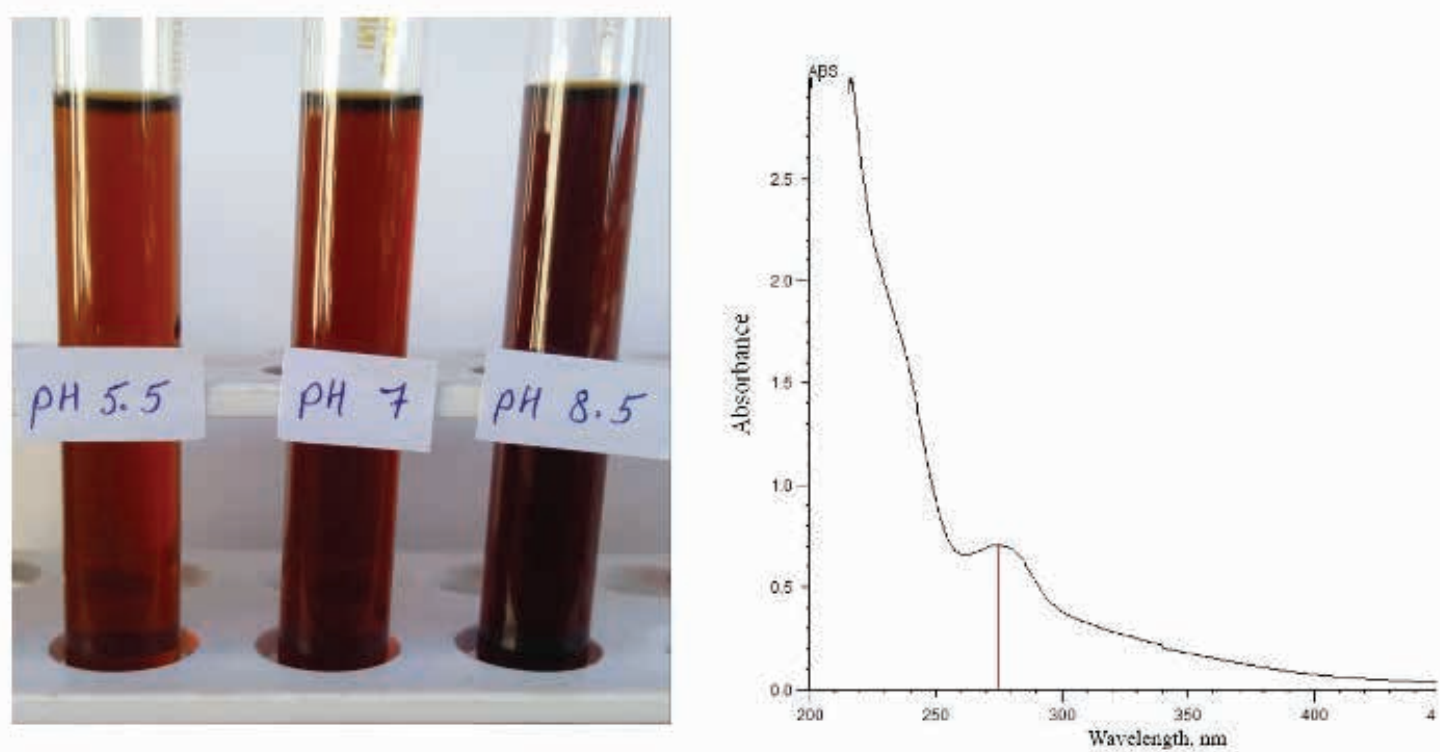

Figure 1. (a) Color of LS solution at different pH. (b) UV-Vis spectra of LS solution. LS, lignosulfonate. 
Table 1. Process parameters and their levels.

\begin{tabular}{|c|c|c|c|c|}
\hline Name of the parameter & Label & Low level (-1) & Medium level (0) & High level (+1) \\
\hline LS $(\%)$ & A & 10 & 15 & 20 \\
\hline Temperature $\left({ }^{\circ} \mathrm{C}\right)$ & B & 60 & 75 & 90 \\
\hline $\mathrm{pH}$ & $\mathrm{C}$ & 5.5 & 7.0 & 8.5 \\
\hline
\end{tabular}

LS, lignosulfonate.

The process parameters were optimized during the application of LS by a $3^{3}$ Box-Behnken design response surface design to obtain the best results. The Box-Behnken design of the experiment is widely used for the optimization of chemical processes as a rotatable design. In the design, the experimental runs are formulated in such a way that no run has all parameters in their extreme levels. As a result, the harsh conditions of the chemical treatment can be avoided and optimum levels of the treatment conditions can be identified using response surface methodology. Experimental design software (DesignExpert version 6.0.8) was used for the design of test runs and statistical evaluation of responses.

\subsection{Characterization}

The FTIR spectra of untreated and LS-treated nylon samples were recorded using a Bruker Alfa FTIR with a scan rate of $4 \mathrm{~cm}^{-1}$ in the wave range of $4,000-500 \mathrm{~cm}^{-1}$. The surface topography of control and LS-treated fabric samples was scanned by Sigma field emission scanning electron microscope from Carl Zeiss.300. Gold coating of samples was done by using a sputter coating set-up. The EDX study of control and LS-treated fabric samples was performed on the same FESEM instrument with EDX attachment. Thermal stability of the LS coated nylon fabrics was investigated by a Perkin Elmer 4000 TGA instrument in a temperature range of $40^{\circ}-400^{\circ} \mathrm{C}$ with a heating arte of $10^{\circ} \mathrm{C}$ per min.

\section{$\underline{2.5}$ Measurement of UPF, K/S, luster, and tensile properties}

The UPF of the fabric samples was estimated with Gester GT-C30 UPF tester, according to AS/NZS4399 standards against the radiation range of $290-400 \mathrm{~nm}$. Ten samples of $2.5 \mathrm{~cm} \times 2.5 \mathrm{~cm}$ were prepared and ten observations were taken from each sample. The color values of the LS-treated samples were evaluated with the aid of the Computer Color Matching system (Konica Minolta, model No. 4808) enabled with JAY pack software. The Gloss Meter (CHN Spec technology, Hangzhou model No. CS300S) was used to analyze the lustrous appearance of fabric samples by estimating the amount of reflected light at an angle of $60^{\circ}$. Tensile strength and elongation of untreated and LS-treated fabric samples were measured by an Instron 4200 tester following ASTM standard.

\subsection{Analysis of fastness properties}

Q-Sun weather-o-meter was used to evaluate the light fastness property of fabric samples as per ISO 105-B02. The fabric samples were exposed for $10 \mathrm{~h}$ by keeping irradiance $-1.10 \mathrm{~W} \times \mathrm{m}^{-2}$, wavelength $-420 \mathrm{~nm}$, and the temperature of the chamber $-50^{\circ} \mathrm{C}$. The washing fastness and color staining were as per ISO 2.

\section{Results and discussion}

\subsection{Optimization of process parameters of LS treatment on nylon fabric for UPF}

Response surface methodology was used to establish an empirical relationship between the response variables, UPF, and a set of experimental factors. The process parameters such as concentration, temperature, and $\mathrm{pH}$ of LS solutions were optimized using $3^{3}$ Box-Behnken response surface designs. The concentration of LS solution was kept at $10 \%$ $(-1), 15 \%(0)$, and $20 \%(+1)$ level, temperature of LS solution was kept at $60{ }^{\circ} \mathrm{C}(-1), 75^{\circ} \mathrm{C}(0)$, and $90{ }^{\circ} \mathrm{C}(+1)$, and $\mathrm{pH}$ of LS solution was kept at $5.5(-1), 7.0(0)$, and $8.5(+1)$ respectively. According to this design, a total of 17 samples were prepared. UPF-values of all these 17 fabric samples are measured and reported in Table 2.

Table 2. Responses of process parameters on UPF.

\begin{tabular}{|c|c|c|c|c|}
\hline Sample & LS (\%) & $\begin{array}{c}\text { Temp. } \\
\left({ }^{\circ} \mathbf{C}\right)\end{array}$ & $\mathbf{p H}$ & UPF \\
\hline 1 & $\mathrm{~A}$ & $\mathrm{~B}$ & $\mathbf{C}$ & \\
\hline 2 & 15 & 75 & 7.0 & 54.68 \\
\hline 3 & 15 & 75 & 7.0 & 54.61 \\
\hline 4 & 20 & 60 & 7.0 & 55.62 \\
\hline 5 & 20 & 75 & 5.5 & 62.13 \\
\hline 6 & 15 & 60 & 5.5 & 42.59 \\
\hline 7 & 10 & 90 & 7.0 & 39.43 \\
\hline 8 & 15 & 90 & 8.5 & 42.2 \\
\hline 9 & 20 & 75 & 8.5 & 37.85 \\
\hline 10 & 10 & 75 & 5.5 & 40.13 \\
\hline 11 & 15 & 60 & 8.5 & 43.14 \\
\hline 12 & 20 & 75 & 8.5 & 45.6 \\
\hline 13 & 10 & 60 & 7.0 & 44.87 \\
\hline 14 & 15 & 75 & 7.0 & 54.73 \\
\hline 15 & 15 & 75 & 7.0 & 55.45 \\
\hline 16 & 15 & 90 & 5.5 & 52.06 \\
\hline 17 & 15 & 75 & 7.0 & 54.08 \\
\hline
\end{tabular}

LS, lignosulfonate; UPF, ultraviolet protection factor. 


\subsubsection{Effect of concentration of LS solution and temperature on UPF}

Figure 2 shows the contour and response surface plot between concentration and temperature of LS solution on fabric UPF. The contour plot shows that UPF increases with an increase in the concentration of the LS solution. UPF initially increases and then decreases with an increase in treatment temperature. The maximum value of UPF is observed at the highest concentration of LS solution (20\%) around the mid-value of the treatment temperature $\left(75^{\circ} \mathrm{C}\right)$. The increase in UPF with an increase in the concentration of LS solution is attributed to an increase in the add-on of lignin on fabric. Earlier studies have also verified that lignin is a good absorber of UVR [15].

\subsubsection{Effect of temperature and $\mathrm{pH}$ on UPF}

The contour and response surface plot of UPF factor against temperature and $\mathrm{pH}$ of LS solution is shown in Figure 3. It can be seen from the contour diagram that the UPF factor initially marginally increases and then decreases with an increase in temperature and $\mathrm{pH}$ of LS solution. It is observed from Table 2 that the highest UPF is observed with Treatment 4 . (at $75^{\circ} \mathrm{C}$ temperature and $5.5 \mathrm{pH}$ ). From the table, it is ascertained that the $\mathrm{pH}$ of the LS solution is an important factor in determining the UPF. The UPF-values were found to be higher in acidic $\mathrm{pH}$, in comparison with alkaline $\mathrm{pH}$.

\subsubsection{Effect of LS concentration and pH on UPF}

Figure 4 shows the contour and response surface plot of the influence of concentration and $\mathrm{pH}$ of LS solution on UPF. The contour plot clearly indicates that UPF increases with an increase in the concentration of LS solution. It initially increases then decreases with an increase in the $\mathrm{pH}$ of the solution. The increase in UPF with an increase in the concentration of LS solution is attributed to increasing lignin add-on on fabric, leading to an increase in the ability to absorb the UVR. It is also indicated that an acidic medium leads to an increase in fabric UPF rather than an alkali medium.

\subsubsection{ANOVA Analysis - UPF}

The value of UPF depends on process parameters. The main effects and interaction effects of the process parameters on UPF are shown in ANOVA Table 3. The Model F-value of 4.62


Figure 2. Effect of temperature and concentration of LS on UPF of nylon fabric. LS, lignosulfonate; UPF, ultraviolet protection factor.
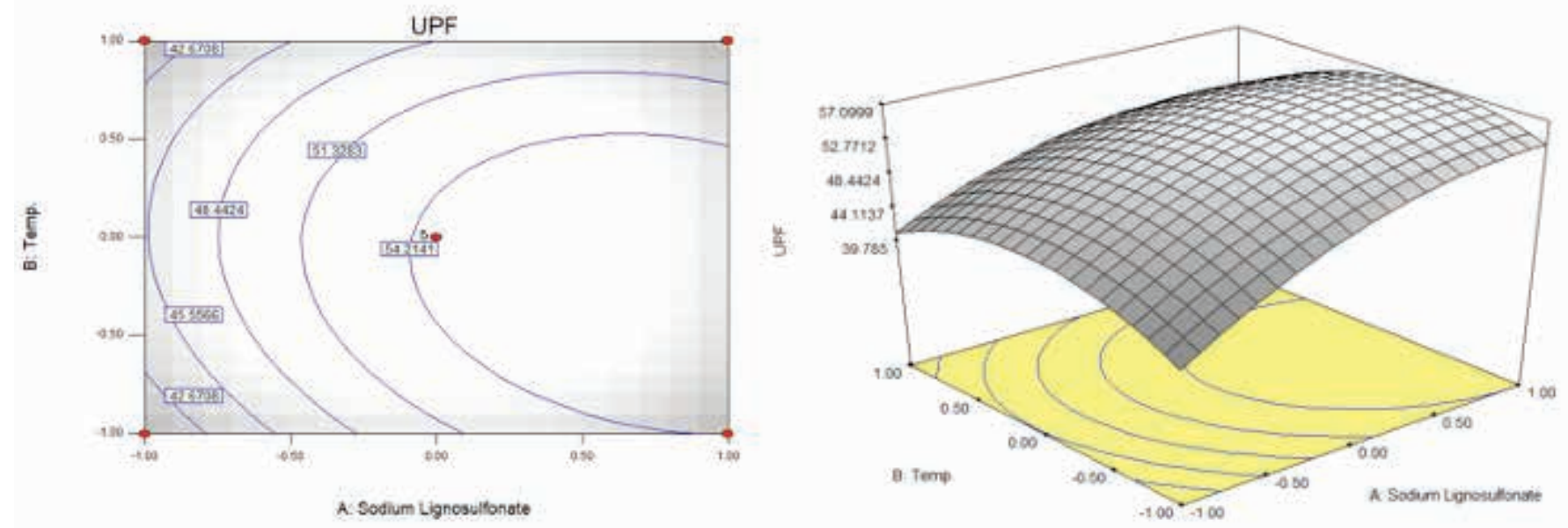

Figure 3. Effect of temperature and $\mathrm{pH}$ on UPF of nylon fabric. UPF, ultraviolet protection factor. 

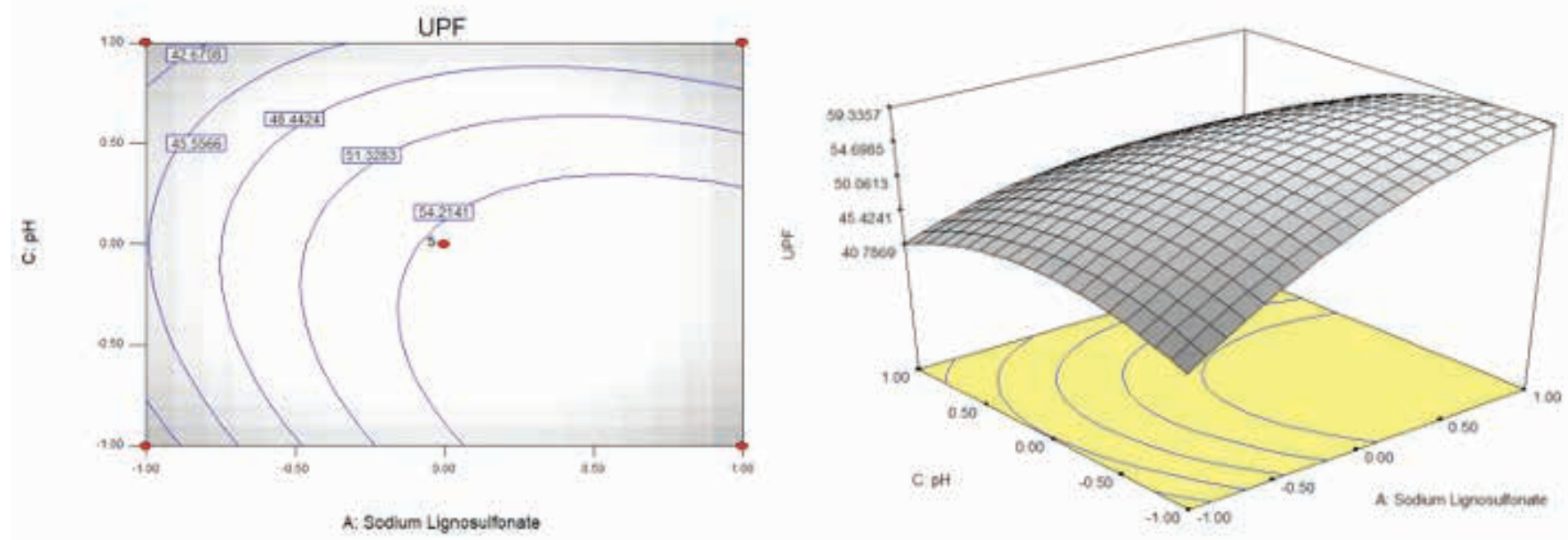

Figure 4. Effect of (a) pH and (b) concentration of LS on UPF of nylon fabric. LS, lignosulfonate; UPF, ultraviolet protection factor.

implies the model is significant. There is only a $2.80 \%$ chance that a "Model F-value" this large could occur due to noise. Values of "Prob > F" less than 0.05 indicate model terms are significant. In this case, $A, C, B^{2}$ are significant model terms. Among the main effects, LS concentration (A) is the most significant factor. It depicted that the presence of sodium LS on nylon fabric is substantially enhancing the ultraviolet protection of nylon fabric. The UPF of nylon fabric, when treated with $20 \%$ LS concentration, achieved up to 62.13 in comparison to 10.96 for the control untreated nylon sample. The UPF-value of fabric is in an excellent rating and a very well safer range of ultraviolet protection. This significant enhancement in UPF is due to the presence of lignin, which contains an aromatic ring, methoxyl $-\mathrm{OCH}_{3}$, Carbonyl $-\mathrm{CO}$, and hydroxyl-OH groups and is known for its ability to absorb ultraviolet rays [11-12].
The model equation in the coded unit is shown in Eq. (1). It can be seen that the fabric UPF is having a quadratic relationship with concentration, temperature, and $\mathrm{pH}$ of LS solution.

$Y(U P F)=54.71+5.59 A-1.11 B-3.52 C-3.69 A^{2}-5.12 B^{2}-4.59 C^{2}-$ 1.63AB- 3.56AC-2.60BC

This equation can well predict the UPF-value with process parameters with a coefficient of determination $\left(R^{2}\right)$ of 0.8559 . This shows the goodness-of-fit of the model. The model is quite efficient as a tool with which to predict the UPF of LS-treated nylon fabric prepared with known process parameters.

Table 3. ANOVA analysis of UPF.

\begin{tabular}{|c|c|c|c|c|c|}
\hline Source & Sum of squares & DF & Mean square & F value & Prob > F \\
\hline Model & 733.37 & 9 & 81.48 & 4.61 & 0.028 \\
\hline$A$ & 250.26 & 1 & 250.26 & 14.18 & 0.007 \\
\hline B & 9.82 & 1 & 9.82 & 0.55 & 0.479 \\
\hline C & 98.84 & 1 & 98.84 & 5.60 & 0.049 \\
\hline$A^{2}$ & 57.35 & 1 & 57.35 & 3.25 & 0.114 \\
\hline$B^{2}$ & 110.40 & 1 & 110.40 & 6.25 & 0.040 \\
\hline $\mathrm{C}^{2}$ & 88.78 & 1 & 88.78 & 5.033 & 0.059 \\
\hline$A B$ & 10.61 & 1 & 10.61 & 0.60 & 0.463 \\
\hline$A C$ & 50.76 & 1 & 50.76 & 2.87 & 0.133 \\
\hline$B C$ & 27.09 & 1 & 27.092 & 1.53 & 0.255 \\
\hline Residual & 123.46 & 7 & 17.63 & - & - \\
\hline Lack of fit & 122.50 & 3 & 40.83 & 170.89 & 0.0001 \\
\hline Pure error & 0.95 & 4 & 0.23 & - & - \\
\hline Corr total & 856.83 & 16 & - & - & - \\
\hline
\end{tabular}




\subsection{Mechanism of reaction between LS and nylon}

The proposed mechanism of reaction between nylon molecule with sodium lignosulphonate is shown in Figure 5. Nylon 6 and sodium lignosulphonate molecules possess $-\mathrm{NH}$ - and -free radical group $-\mathrm{OH}-$ respectively. Free radical of $\mathrm{OH}$ group of LS attaches with $-\mathrm{NH}-$ group of nylon 6 , consequently releasing $\mathrm{H}_{2} \mathrm{O}$ molecule during reaction.
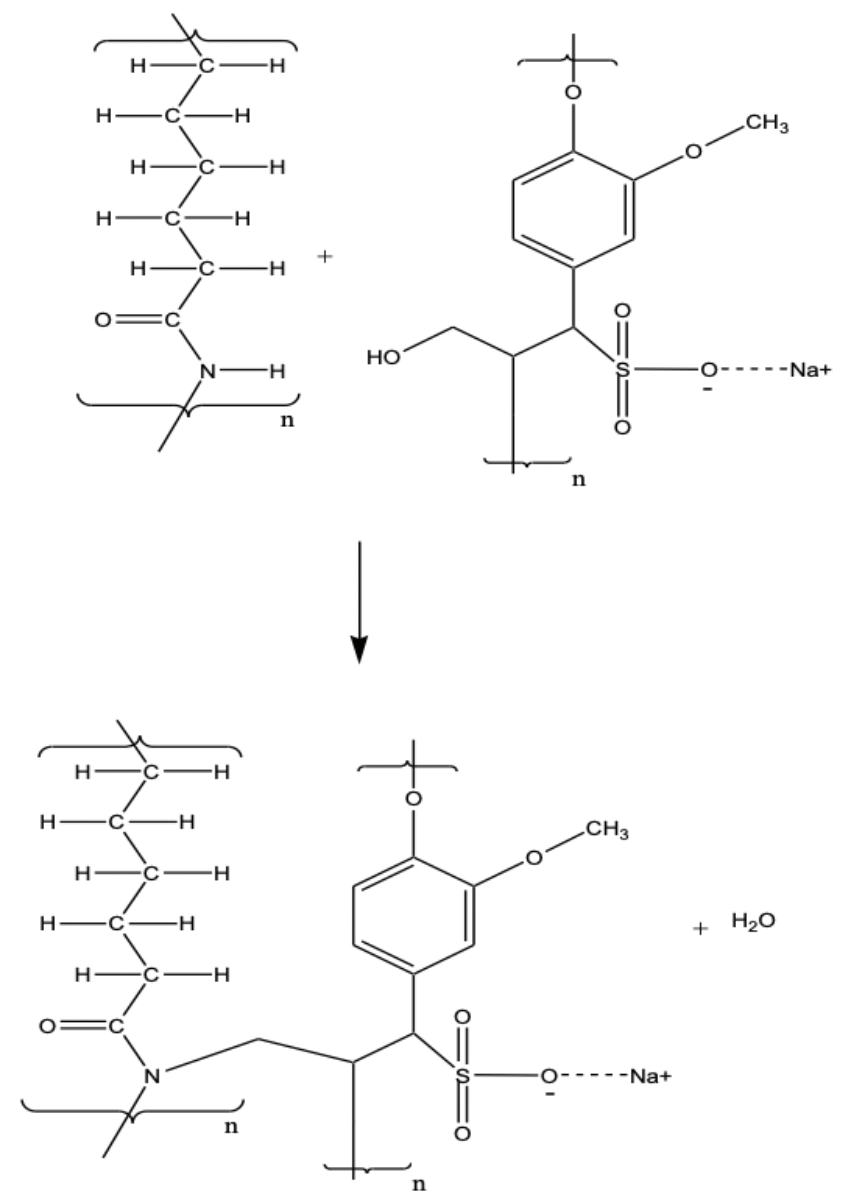

Figure 5. Reaction mechanism of LS with Nylon 6. LS, lignosulfonate.

\section{$\underline{\text { 3.3 FTIR Analysis }}$}

FTIR spectra of uncoated nylon and LS coated nylon are shown in Figure 6. FTIR spectra of pure nylon fiber shows absorption band at $3,297 \mathrm{~cm}^{-1}$ that may be attributed to the vibration of $-\mathrm{OH}$ groups, band at $2,922 \mathrm{~cm}^{-1}$ that may be attributed to $\mathrm{CH}$ asymmetric stretching, band at $1,635 \mathrm{~cm}^{-1}$ that may be attributed to amide I band, band at $1,537 \mathrm{~cm}^{-1}$ that may be attributed to $-\mathrm{CH}_{2}$ asymmetric deformation of amide II, band at $1,462 \mathrm{~cm}^{-1}$ that may be attributed to $-\mathrm{CH}_{2}$ scissoring and $-\mathrm{NH}$ deformation, and band at $1,369 \mathrm{~cm}^{-1}$ that may be attributed to $\mathrm{CH}_{2}$ wagging of amide III. The FTIR spectra of LS-treated nylon shows all vital peaks of nylon fiber as well as characteristic peaks of lignin. Shifting of the peak of $1,635 \mathrm{~cm}^{-1}$ of nylon to a new position at $1,590 \mathrm{~cm}^{-1}$ after LS treatment indicates the intermolecular interaction between $-\mathrm{NH}-$ group of nylon with $-\mathrm{OH}$ group of lignin [16]. The prominent peak at $1,024 \mathrm{~cm}^{-1}$ indicates $\mathrm{C}-\mathrm{H}$ Plane deformation of lignin $[17,18]$.

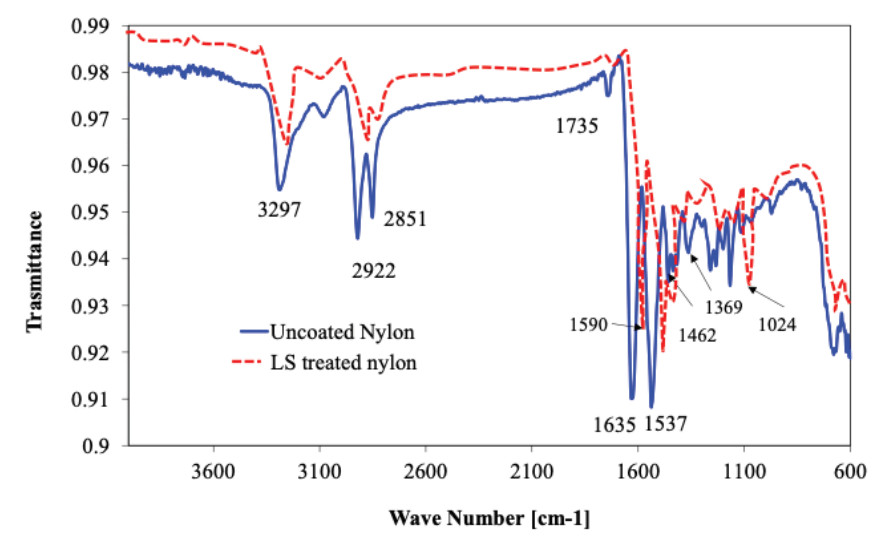

Figure 6. FTIR spectra of untreated and LS-treated nylon. LS, lignosulfonate.

\subsection{FESEM and EDX analysis}

The surface topographic FESEM images of control and LStreated nylon 6 fabric samples are shown in Figure 7. It is evident from the figure that there is no prominent deposition which is visible on the fiber surface. This may be because of the chemical reaction between the nylon and LS, rather than a physical coating on the surface.

For evidencing the presence of LS on the surface of nylon, EDX analysis was carried out for all samples. The EDX spectra of untreated and $20 \%$ LS-treated nylon fabric are shown in Figure 8. It is visible from EDX analysis that the control nylon fabric showed three peaks for carbon, nitrogen, and oxygen elements. In addition to the said peaks, the LS-treated fabric sample showed extra peaks for sulfur and sodium, due to LS treatment. From Table 4, it is observed that the pure nylon fabric sample has $64.78 \%$ carbon, $17.56 \%$ nitrogen, and $17.65 \%$ oxygen. The element-composition is changed after LS treatment on nylon fabric in terms of sodium and sulfur. The LS-treated nylon fabric sample has $63.87 \%$ carbon, $16.77 \%$ nitrogen, $19.04 \%$ oxygen, $0.24 \%$ sodium, and $0.07 \%$ sulfur.

\subsection{Color Strength of LS-treated fabrics}

The fabric color strength of control and LS-treated fabric at different concentrations are measured and the results are shown in Table 5. Lignin is colored brown due to the presence of chromophoric groups such as catechols, aromatic ketones, coniferaldehyde, stilbenes, and conjugated phenolics, [19, 20]. These chromophores can interact with nylon to impart a yellow color to the fabric (Figure 9). Table 5 demonstrates that the increase in the concentration of sodium LS drives the increase in the value of color strength linearly. These increases in color-strength values of LS-treated fabric samples were found to be statistically significant, as proved by the ANOVA test. It is reported that the UPF of the fabric harbors a positive relationship with the color values of the fabric, because a dark color absorbs higher UV radiation than light shades [21]. In our study also, we have observed a direct positive correlation between the UPF and K/S. 


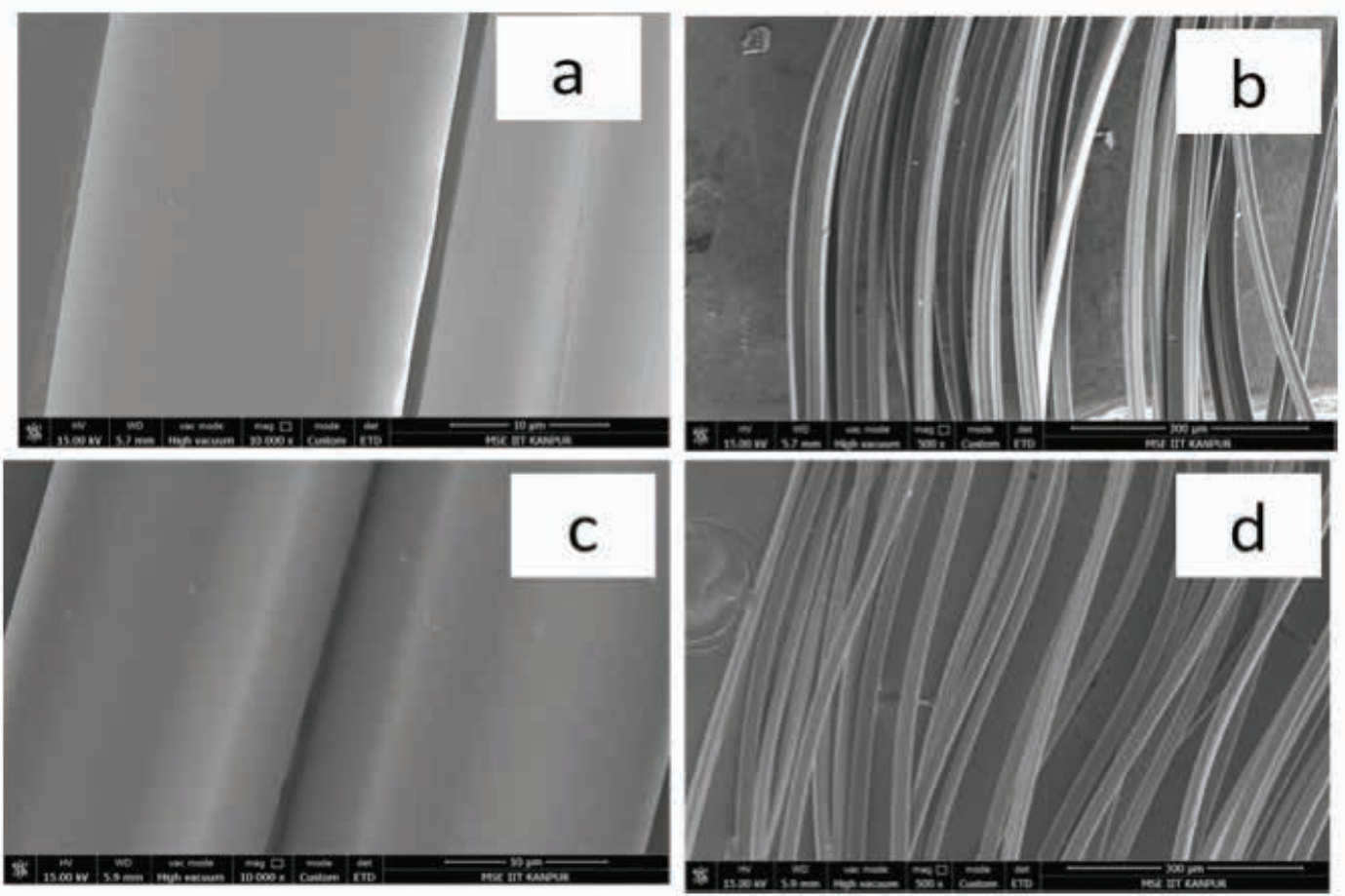

Figure 7. SEM Images of (a) LS coated Nylon (1,000x), (b) LS coated Nylon (500x)

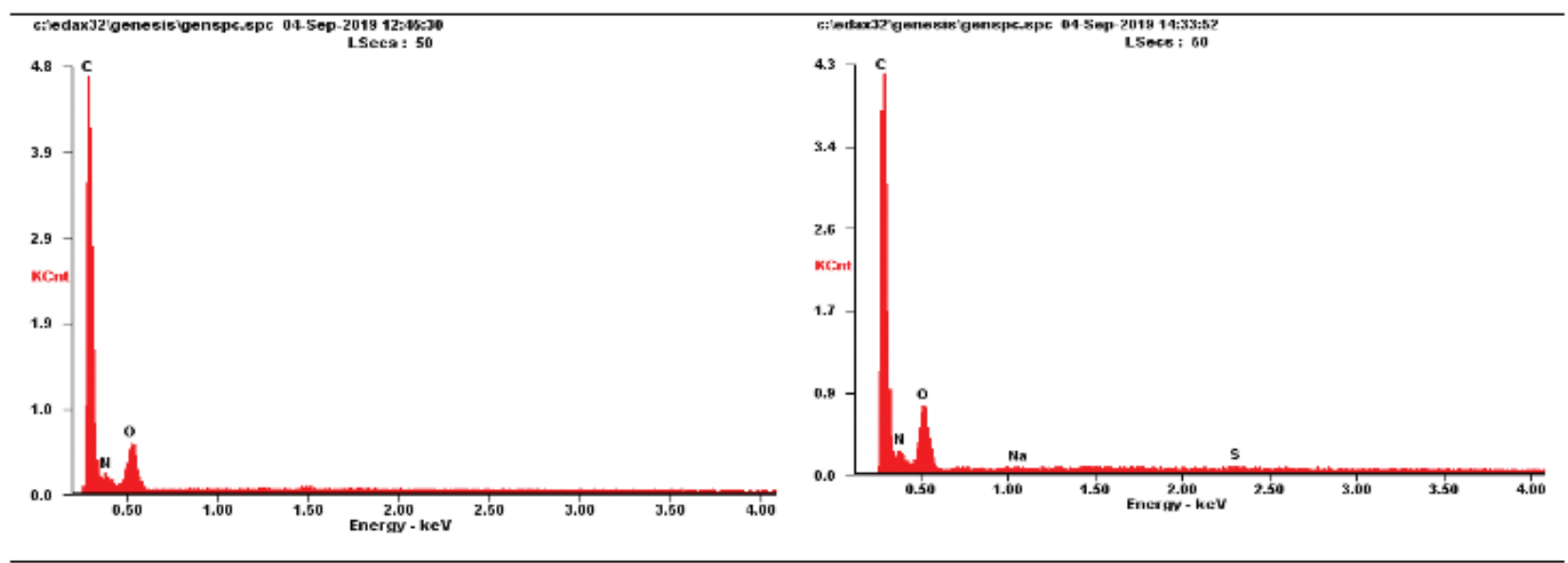

Figure 8. EDX spectra of (a) control Nylon and (b) LS-treated Nylon samples. EDX, energy dispersive x-ray; LS, lignosulfonate.

Table 4. EDX analysis of control and LS-treated nylon samples.

\begin{tabular}{|c|c|c|c|c|c|c|}
\hline Sample & C (A.W\%) & N (A.W\%) & O (A.W\%) & Na (A.W\%) & S (A.W\%) & Total \\
\hline Control nylon & 64.79 & 17.56 & 17.65 & - & - & 100 \\
\hline $20 \%$ LS-treated nylon & 63.87 & 16.79 & 19.04 & 0.24 & 0.07 & 100 \\
\hline
\end{tabular}

EDX, energy dispersive $\mathrm{x}$-ray; LS, lignosulfonate.

Table 5. Colour strength (K/S), luster, washing fastness, and light fastness.

\begin{tabular}{|c|c|c|c|c|c|c|c|c|}
\hline \multirow{2}{*}{$\begin{array}{c}\text { Sample } \\
\text { code }\end{array}$} & $\begin{array}{c}\text { LS con } \\
(\%)\end{array}$ & \multicolumn{4}{|c|}{ Color strength } & \multirow{2}{*}{ Lustre } & $\begin{array}{c}\text { Washing } \\
\text { fastness }\end{array}$ & $\begin{array}{c}\text { Light } \\
\text { fastness }\end{array}$ \\
\cline { 3 - 8 } & & K/S & L & A & B & & 5 & 4 \\
\hline N & 0 & 0.10 & 91.9 & 0.76 & 3.01 & 4.55 & 5 & 4 \\
\hline NLS-10 & 10 & 2.24 & 80.4 & 4.28 & 21.14 & 3.67 & 5 & 4 \\
\hline NLS-15 & 15 & 4.10 & 75.62 & 5.53 & 25.31 & 2.88 & 5 \\
\hline
\end{tabular}

N, nylon, LS, lignosulfonate. 

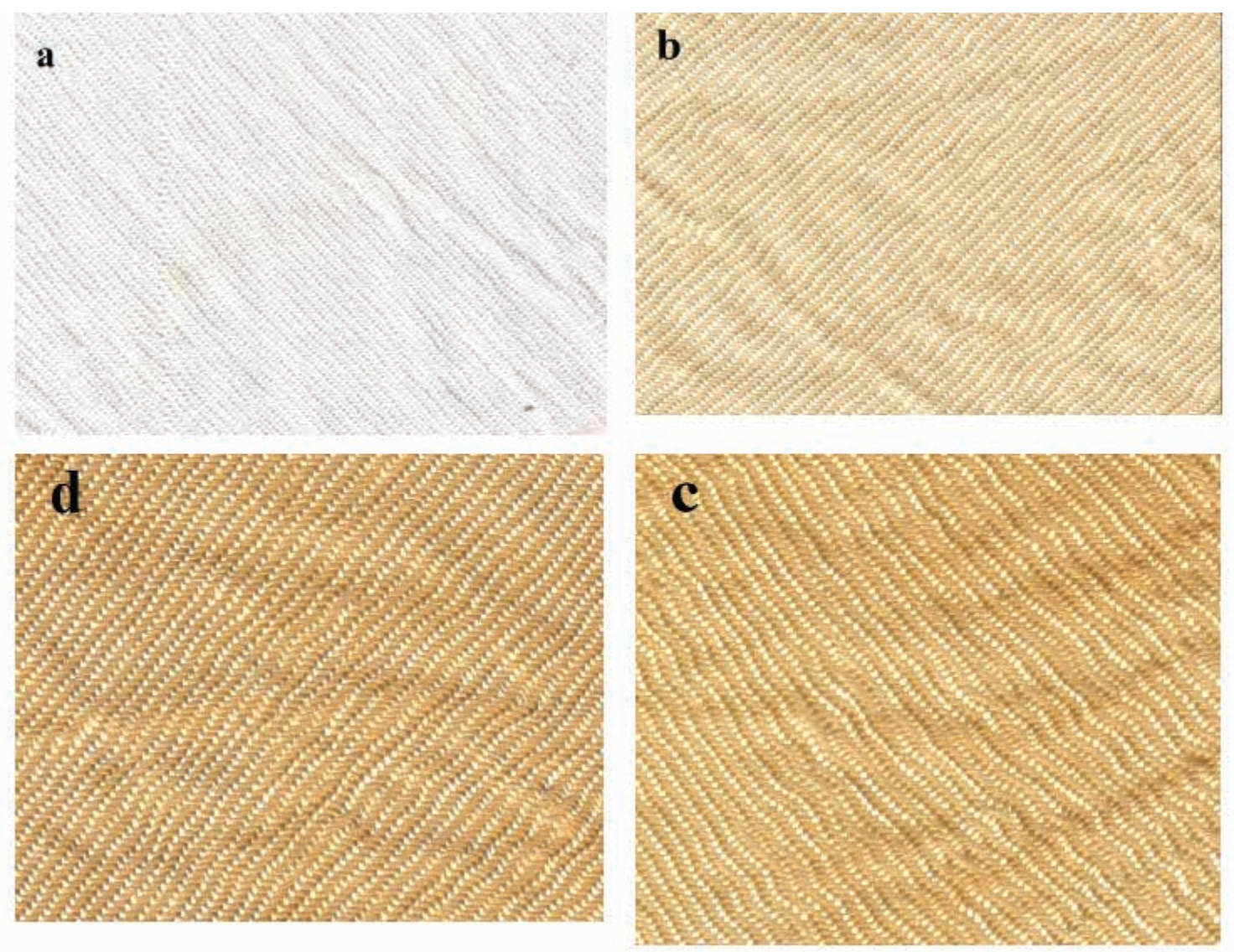

Figure 9. Images of (a) Control, (b) 10\%, 15\%, and 20\% LS-treated nylon fabrics. LS, lignosulfonate.

Table 6. Tensile properties of untreated and LS-treated nylon fabric

\begin{tabular}{|c|c|c|c|c|c|c|c|}
\hline \multirow{2}{*}{$\begin{array}{c}\text { Sample } \\
\text { code }\end{array}$} & \multirow[t]{2}{*}{ LS con (\%) } & \multicolumn{2}{|c|}{ Tensile strength $\left(\mathrm{Nm}^{-2} \times \mathbf{1 0}^{6}\right)$} & \multicolumn{2}{|c|}{ Elongation (mm) } & \multicolumn{2}{|c|}{ Tensile modulus $\left(\mathrm{Nm}^{-2} \times 10^{6}\right)$} \\
\hline & & Mean & $\begin{array}{l}\text { Standard } \\
\text { deviation }\end{array}$ & Mean & $\begin{array}{l}\text { Standard } \\
\text { deviation }\end{array}$ & Mean & $\begin{array}{l}\text { Standard } \\
\text { deviation }\end{array}$ \\
\hline $\mathrm{N}$ & 0 & 62.36 & 2.10 & 35.62 & 1.55 & 277.90 & 22.18 \\
\hline NLS-10 & 10 & 67.29 & 2.51 & 40.54 & 1.35 & 276.18 & 33.37 \\
\hline NLS-15 & 15 & 69.67 & 4.32 & 39.96 & 1.32 & 297.85 & 21.85 \\
\hline NLS-20 & 20 & 67.56 & 1.71 & 39.39 & 1.77 & 300.69 & 9.90 \\
\hline
\end{tabular}

LS, lignosulfonate.

\subsection{Fastness properties of LS-treated fabrics}

The light fastness properties of LS-treated fabric samples tested under the xenon arc lamp are shown in Table 5. All LStreated fabric samples reveal a very good lightfastness rating. It is evident from light fastness data that sodium LS has an excellent affinity with nylon fiber surface under the xenon arc light. The washing fastness of LS-treated nylon samples is excellent and exhibits staining neither on cotton nor on scoured nylon fabric after washing. This may be attributed to the fact that sodium LS has an effective affinity with nylon fabrics. It is also noted that the soaping process given after the LS treatment is one of the reasons for getting good fastness properties.

\subsection{Lustre of LS-treated fabrics}

Fabric luster is one of the attributes which affects the visual appearance of a fabric. It is the amount of specular light the fabric reflects. The luster behavior of control and LS-treated fabrics at different concentrations are shown in Table 5. The untreated nylon fabric was found to be highly lustrous (4.55 Gloss $60^{\circ}$ ). The presence of LS on treated nylon fabric samples suppressed the luster intensity and the concentration of LS is inversely proportional to the luster intensity. The reduction in luster intensity in the presence of sodium LS is attributed to its coloring potential which exhibits higher absorption of light in the visible region. 


\subsection{Tensile properties of untreated and LS-treated fabrics}

Tensile strength, modulus, elongation of untreated nylon fabrics, and all LS-treated nylon fabrics are shown in Table 6. It can be seen that after LS treatment there is a slight improvement in the tensile properties of nylon fabric. Therefore, it can be concluded that the LS treatment is enhancing the strength of the nylon fabrics and is therefore very safe for the modification for bringing about the UV protection properties in it. The enhancement of the tensile strength of nylon fabric after LS treatment is due to the significant intermolecular interaction as shown in Figure 5.

\subsection{TGA of LS-treated nylon samples}

TGA analysis of untreated nylon and $20 \%$-LS-solution-treatednylon-sample was conducted to understand the thermal degradation behavior. The TGA thermogram is shown in Figure 10. It can be seen that LS treatment does not cause any significant deterioration of the thermal stability of nylon. The insignificant weight loss at $400^{\circ} \mathrm{C}$ may be due to the loss of LS molecules from the nylon surface without affecting the nylon 6 polymer.

\section{Conclusion}

The Box-Behnken response surface methodology reveals that LS concentration (A) is the most significant main-effect influencing UPF of the LS-treated nylon fabric. The maximum UPF factor was achieved as 62.13 when LS treatment was carried out with $20 \%$ LS concentration, $75^{\circ} \mathrm{C}$ temperature, and at $5.5 \mathrm{pH}$. It has been observed that maximum UPF can be achieved with a high level of LS concentration, and a medium level of temperature and $\mathrm{pH}$. The chemical reaction between free radical of $\mathrm{OH}$ group of $\mathrm{LS}$ and $-\mathrm{NH}-$ group of nylon 6 has been suspected, which is evidenced by FTIR study. FESEM and EDX results provide the evidence of chemical reaction of LS with nylon fabrics. UV-Vis Spectroscopy results depict the deeper color strength of the samples with concentrated LS treatment. Due to this, the luster of the fabrics reduced when treated with a concentrated solution. Tensile modulus, strength, elongation, and thermal stability of the nylon fabrics do not

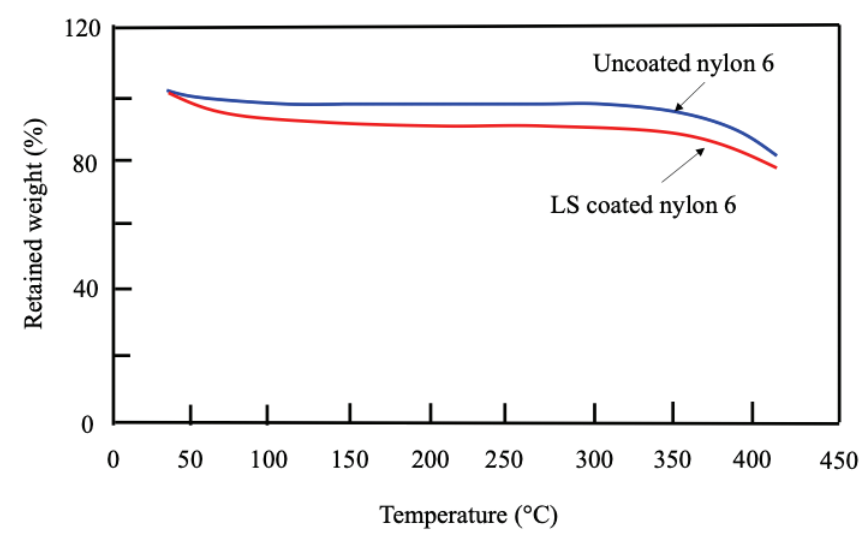

Figure 10. TGA analysis of untreated and LS-treated nylon fabrics. LS, lignosulfonate; TGA, thermo-gravimetric analysis. deteriorate after the LS treatment. The wash fastness of all LStreated fabrics were found to be excellent.

\section{Acknowledgment}

This work was conducted in Uttar Pradesh Textile Technology Institute, Kanpur, and supported by the Technical Education Quality Improvement Program (TEQIP-III) of the World Bank.

\section{References}

[1] Ammayappan, L., Jose, S. (2015). Functional aspects, eco-testing, and environmental impact of natural dyes. Handbook of Sustainable Apparel Production, CRC Press.

[2] Ibrahim, N. A, Allam, E. A., El-Hossamy, M. B., El-Zairy, W. M. (2007). UV-protective finishing of cellulose/wool blended fabrics. Polymer-Plastics Technology and Engineering, 46(9), 905-911.

[3] Pandey, R., Patel, S., Pandit, P., Nachimuthu, S., Jose, S. (2018). Coloration of textiles using roasted peanut skin-an agro processing residue. Journal of Cleaner Production, 172, 1319-1326.

[4] Gies, P. (2007). Photo protection by clothing. Photodermatol Photoimmunol Photomed, 23, 264-274.

[5] Osterwalder, U., Schlenker, W., Rohwer, H. (2000). Facts and fiction on ultraviolet protection by clothing. Radiation Protection Dosimetry, 91(1), 255-259.

[6] Saravanan, D. (2007). UV protection textile Materials. AUTEX Research Journal, 7(1), 53-62.

[7] Fatehi, P., Ni, Y. (2011). Integrated forest biorefinery Sulfite process. In: Zho, J, Zhang, X, Pan, X. (Eds.). Sustainable production of fuels, chemicals and fibers from forest biomass. American Chemical Society (Washington, DC). pp. 409-441.

[8] Vishtal, A. G., Kraslawski, A. (2011). Challenges in industrial applications of technical lignins. BioResources, 6, 3547-3568.

[9] Feng, B., Guo, W., Peng, J., Zhang, W. (2018). Separation of scheelite and calcite using calcium lignosulphonate as depressant. Separation and Purification Technology, 199, 346-350.

[10] Alsulami, Q. A., Albukhari, S. M., Hussein, M. A., Tay, G. S., Rozman, H. D. (2020). Biodegradable lignin as a reactive raw material in UV curable systems. PolymerPlastics Technology and Materials, 59(13), 1387-1406. doi: 10.1080/25740881.2020.1750649.

[11] Abreu, H.S., Nascimento, A.M., Maria, M.A. (1999). Lignin structure and wood properties. Wood and Fiber Science, 31(4), 426-433.

[12] Zimniewska, M., Kozlowski, R., Batog, J. (2008). Molecular Crystals and Liquid Crystals, 484, 44/[416].

[13] Aro, T., Fatehi, P. (2017). Production and application of lignosulfonates and sulfonated lignin. ChemSusChem, 10(9), 1861-1877.

[14] Yang, D., Qui, X., Zhou, M., Lou, H. (2007). Properties of sodium lignosulfonate as dispersant of coal water slurry. Energy Conversion and Management, 48(9), 2433-2438.

[15] Gu, X. D., Sun, M. Y., Zhang, L., Fu, H. W., Cui, L., et al. (2010). UV-B induced changes in the secondary metabolites of morus alba L. leaves. Molecules, 15, 29802993. 
[16] Jose, S., Mishra, L., Basu, G., Samanta, A. K. (2017). Study on reuse of coconut fiber chemical retting bath. Part II - recovery and characterization of lignin. Journal of Natural Fibers, 14, 510-518.

[17] Pandey, R., Jose, S., Basu, G., Sinha, M. K. (2019). Novel methods of degumming and bleaching of indian flax variety tiara. Journal of Natural Fibers, 1-11.

[18] Jose, S., Mishra, L., Debnath, S., Pal, S., Munda, P. K., Basu, G. (2019). Improvement of water quality of remnant from chemical retting of coconut fibre through electrocoagulation and activated carbon treatment. Journal of Cleaner Production, 210, 630-637.
[19] Azad fallah, M., Mirshokraei, S. A., Latibari, A. J. (2008). Photo degradation of acidolysis lignin from BCMP. Molecules, 13, 3129-3139.

[20] Ajao, O., Jeaidi, J., Benali, M., Restrepo, A. M., El. Mehdi, $N$., et al. (2018). Quantification and variability analysis of lignin optical properties for color-dependent industrial applications, Molecules, 23(2), 377.

[21] Jose, S., Pandit, P., Pandey, R. (2019). Chickpea husk - A potential industrial agro residue for the coloration and functional finishing of textiles. Industrial Crops and Products, 142. 111833. 